\title{
A New Model for Selfish Routing*
}

\author{
Thomas Lücking $^{1}$, Marios Mavronicolas ${ }^{2 \star \star}$, Burkhard Monien ${ }^{1}$, and \\ Manuel Rode ${ }^{1}$
}

1 Faculty of Computer Science, Electrical Engineering and Mathematics, University of Paderborn, Fürstenallee 11, 33102 Paderborn, Germany.

$$
\text { \{luck, bm, rode\}@uni-paderborn.de }
$$

2 Department of Computer Science, University of Cyprus, P. O. Box 20537, Nicosia

CY-1678, Cyprus. mavronic@ucy.ac.cy

\begin{abstract}
In this work, we introduce and study a new model for selfish routing over non-cooperative networks that combines features from the two such best studied models, namely the KP model and the Wardrop model in an interesting way.

We consider a set of $n$ users, each using a mixed strategy to ship its unsplittable traffic over a network consisting of $m$ parallel links. In a Nash equilibrium, no user can increase its Individual Cost by unilaterally deviating from its strategy. To evaluate the performance of such Nash equilibria, we introduce Quadratic Social Cost as a certain sum of Individual Costs - namely, the sum of the expectations of the squares of the incurred link latencies. This definition is unlike the KP model, where Maximum Social Cost has been defined as the maximum of Individual Costs.

We analyse the impact of our modeling assumptions on the computation of Quadratic Social Cost, on the structure of worst-case Nash equilibria, and on bounds on the Quadratic Coordination Ratio.
\end{abstract}

\section{Introduction}

\subsection{Motivation and Framework}

Nash Equilibria and Outline. Nash equilibrium 2324 is arguably the most robust equilibrium concept in (non-cooperative) Game Theory 1 At a Nash equilibrium, no player of a strategic game can unilaterally improve its objective by switching to a different strategy. In a pure Nash equilibrium, each player chooses exactly one strategy (with probability one); in a mixed Nash equilibrium, the choices of each player are modeled by a probability distribution over strategies. Of special interest to our work is the fully mixed Nash equilibrium [22], where

* This work has been partially supported by the IST Program of the European Union under contract numbers IST-1999-14186 (ALCOM-FT) and IST-2001-33116 (FLAGS), and by research funds at University of Cyprus.

${ }^{\star}$ Part of the work of this author was performed while visiting Faculty of Computer Science, Electrical Engineering and Mathematics, University of Paderborn.

${ }^{1}$ See [25] for a concise introduction to contemporary Game Theory. 
each user chooses each strategy with non-zero probability. Nash equilibria have some very nice properties; most notably, for finite games, there always exists a mixed Nash equilibrium 24.

In this work, we embark on a systematic study, within a new model for selfish routing over non-cooperative networks that we introduce, of some interesting algorithmic and mathematical properties of Nash equilibria for some specific routing game formulated in this context. Our new model for selfish routing is an interesting hybridization of the two most famous models for selfish routing that were studied in the literature before; these are the so called KP model [19] and Wardrop model 430.

The KP Model and the Wardrop Model. The KP and the Wardrop models differ with respect to the assumptions they are making about: 1 . the structure of the routing network; 2 . the splittability or unsplittability of the users' traffics; 3 . the definition of Individual Cost for a user they use for defining Nash equilibria; 4. the type of Nash equilibria (pure or mixed) they consider; 5 . the specific definitions they employ for Social Cost, a performance measure for Nash equilibria, and for Social Optimum, an optimality measure for traffic assignments (not necessarily equilibria). The definitions for Social Cost usually relate to Individual Costs. In either model, these two definitions give rise to Coordination Ratio, the maximum value of the ratio of Social Cost over Social Optimum; a worst-case Nash equilibrium is one that maximizes its particular Social Cost.

In the KP model, a collection of $n$ users is assumed; each user employs a mixed strategy, which is a probability distribution over $m$ parallel links, to control the shipping of its own assigned traffic. In the KP model, traffics are unsplittable. For each link, a capacity specifies the rate at which the link processes traffic. Allowing link capacities to vary arbitrarily gives rise to the standard model of related links. A special case of the model of related links is the model of identical links, where all link capacities are identical. Reciprocally, in the model of identical users, all user traffics are equal; they may vary arbitrarily in the model of arbitrary users. In a Nash equilibrium, each user selfishly routes its traffic on those links that minimize its Individual Cost: its expected latency cost on that link, given the network congestion caused by the other users. In the KP model, the Social Cost of a Nash Equilibrium, henceforth called Maximum Social Cost, is the expectation, over all random choices of the users, of the maximum, over all links, latency through a link; the Social Optimum, henceforth called the Maximum Social Optimum, is the least possible maximum, over all links, latency through a link that could be attained had global regulation been available; correspondingly, the Coordination Ratio in the KP model will henceforth be called the Maximum Coordination Ratio. It follows that the Maximum Social Cost in the KP model is the maximum of Individual Costs.

In the Wardrop model 1130, there have been considered arbitrary networks with latency functions for edges. Moreover, the traffics are splittable into arbitrary pieces. Here, unregulated traffic is modeled as a network flow. Equilibrium flows have been classified as flows with all flow paths used between a given pair of a source and a destination having the same latency. Equilibrium flows are optimal solutions to a convex program, in case the edge latency functions are convex. An equilibrium in this model can be interpreted as a Nash equilibrium 
in a game with infinitely many users, each carrying an infinitesimal amount of traffic from a source to a destination. Thus, the Wardrop model restricts to pure Nash equilibria. The Individual Cost of a user is defined as the sum of the edge latencies on a path from the user's source to its destination. The Social Cost of a Nash equilibrium is the sum of all Individual Costs. The Social Optimum is the least possible, over all network flows, sum of Individual Costs.

The New Model for Selfish Routing. Our new model for selfish routing over non-cooperative networks is a hybridization of the KP model [19] and the Wardrop model [1130. More specifically, we follow the KP model to consider the simple parallel links network (which, however, is also a special case for the Wardrop model). We also follow the KP model to consider unsplittable traffics and mixed Nash equilibria. The Individual Cost we adopt is also identical to that adopted in the KP model - the expected latency cost on a link. However, we follow the Wardrop model to model Social Cost as a certain sum of Individual Costs, which we will later describe. In some sense, our new model is the Wardrop model restricted to the simple parallel links network but modified to allow for unsplittable traffics and for mixed strategies; these two features were borrowed from the KP model. Our work is the first step toward accommodating unsplittable traffics within the Wardrop model.

For any link, consider the square of the traffic through the link divided by the link capacity; taking the expectation of this and adding up over all links yields the Social Cost for our model. Call it Quadratic Social Cost. In correspondence with Quadratic Social Cost, we also define and study in our new model Quadratic Optimum and Quadratic Coordination Ratio. Naturally, the former is the least possible sum of the squares of total traffic through a link divided by the link capacity; the latter is the maximum value of the ratio of Quadratic Social Cost over Quadratic Social Optimum. Since Nash equilibria are defined with respect to Individual Costs (but are independent of Social Cost), the Nash equilibria in our new model coincide with those in the KP-model since the two adopt the same Individual Costs.

Note that the commutativity between expectation and sum in the definition of Quadratic Social Cost has been unavailable (between expectation and maximum) in the definition of Maximum Social Cost for the KP model. So, this commutativity allows hopes for some more tractable analysis of several problems regarding some interesting algorithmic, combinatorial, structural and optimality properties of Nash equilibria in the new model.

\subsection{Contribution and Significance}

We partition our results into three major groups.

Combinatorial Expressions for Quadratic Social Cost. In the most general model of arbitrary users and arbitrary links, we obtain an elegant, recursive combinatorial formula for Quadratic Social Cost, implying a dynamic programming algorithm to compute Quadratic Social Cost. Furthermore, we derive simple, combinatorial expressions for the Quadratic Social Cost of the fully mixed Nash equilibrium in case of arbitrary users and identical links, and identical users and arbitrary links, respectively. 
The Worst-case Nash Equilibrium. A natural problem that arises in the context of Quadratic Social Cost is to identify the worst-case Nash equilibrium the one that maximizes, for each specific choice of user traffics and link capacities, the Quadratic Social Cost. We address this problem in the particular setting of the model of identical users and identical links, where the fully mixed Nash equilibrium always exists. We prove that, in this particular setting, the worstcase Nash equilibrium is the fully mixed Nash equilibrium.

Bounds on Quadratic Coordination Ratio. For the model of arbitrary users and identical links we prove that the Quadratic Coordination Ratio for pure Nash equilibria is precisely $\frac{9}{8}$. In case of identical users and related links, we discover that the Quadratic Coordination Ratio for pure Nash equilibria increases slightly to $\frac{4}{3}$. We next turn to the model of arbitrary users and identical links. Here, we restrict ourselves to the fully mixed Nash equilibrium. For this setting, we prove an upper bound of $2-\frac{1}{m}$ on Quadratic Coordination Ratio. For identical users the Quadratic Social Cost of the fully mixed Nash equilibrium slightly drops to $1+\min \left\{\frac{m-1}{n}, \frac{n-1}{m}\right\}$ times the optimal Quadratic Social Cost. Since in this setting the fully mixed Nash equilibrium is the worst-case Nash equilibrium, this bound holds for the Quadratic Coordination Ratio.

\subsection{Related Work and Comparison}

The KP model was first introduced in the work of Koutsoupias and Papadimitriou 19]; it was further studied in 91012/14 16 17 18/2122. Fully mixed Nash equilibria were introduced and analyzed in [22. Bounds on Maximum Coordination Ratio were proved in 914,1822. The works by Fotakis et al. 16], by Gairing et al. 17, and by Lücking et al. 21. dwelved into the combinatorial structure and the computational complexity of Nash equilibria for the KP model. In particular, the Fully Mixed Nash Equilibrium Conjecture was motivated by some results in [16], explicitly formulated in [17] and further studied in [21]. The Wardrop model was defined in [30] and further studied in [3,4 11]. Recent studies of selfish routing within the Wardrop model include [27,28,29].

Fotakis et al. [16, Theorem 8] proved that computing the Maximum Social Cost of an arbitrary Nash equilibrium is a $\# \mathcal{P}$-complete problem. This hardness result stands in very sharp contrast to our general, pseudopolynomial algorithm to compute Quadratic Social Cost (Theorem 1).

For the KP model, there are known bounds on Maximum Coordination Ratio of $\Theta\left(\frac{\lg m}{\lg \lg m}\right)$ for the model of arbitrary users and identical links $[9$ 18|1922], of $\Theta\left(\frac{\lg m}{\lg \lg \lg m}\right)$ for the model of arbitrary users and related links [9], and of $O(\sqrt{m})$ for the model of arbitrary users and related links and for pure Nash equilibria [14, which improves the previous bound for small values of $m$. Some of these super-constant bounds stand in very sharp contrast to some of the constant bounds (independent of $m$ and $n$ ) on Quadratic Coordination Ratio we prove in this work. However, for the Wardrop model, there have been shown constant bounds on Coordination Ratio 2728 29].

Other works that have studied Coordination Ratio include [13] for a network creation game and [2] for a network design game. For a survey of recent work 
on selfish routing in non-cooperative networks, see [15]. Work in the scheduling literature that has considered quadratic cost functions for makespan includes [1, 6820 ; work in the networking literature that has considered quadratic cost functions for network delay includes [7].

\subsection{Road Map}

The rest of this paper is organized as follows. Section 2 presents our definitions and some preliminaries. The Quadratic Social Cost of Nash equilibria is studied in Section 3. Section 4 proves that the fully mixed Nash equilibrium maximizes Quadratic Social Cost in the model of identical users and identical links. Our bounds on Quadratic Coordination Ratio are presented in Section 5. Due to lack of space the proofs are omitted. They can be found in the full version.

\section{Framework}

\subsection{Mathematical Preliminaries and Notation}

Throughout, denote for any integer $m \geq 2,[m]=\{1, \ldots, m\}$. For a random variable $X$, denote $\mathcal{E}(X)$ the expectation of $X$. We continue to prove a simple combinatorial inequality.

Lemma 1. For any $k, a, b \in \mathbb{N}$ with $0<k \leq a \leq b, \quad \frac{1}{a^{k}}\left(\begin{array}{l}a \\ k\end{array}\right) \leq \frac{1}{b^{k}}\left(\begin{array}{l}b \\ k\end{array}\right)$.

Finally, we prove a combinatorial lemma that will be useful in a later proof.

Lemma 2. Fix any real number a, where $0<a<1$, and positive integer $r$, and set $A=\frac{r}{a}$. Then, $\sum_{1 \leq k \leq r}\left(\begin{array}{l}r \\ k\end{array}\right) k^{2}\left(\frac{1}{a}\right)^{k}\left(1-\frac{1}{a}\right)^{r-k}=A+\frac{r-1}{r} A^{2}$.

\subsection{General}

We consider a network consisting of a set of $m$ parallel links $1,2, \ldots, m$ from a source node to a destination node. Each of $n$ network users $1,2, \ldots, n$, or users for short, wishes to route a particular amount of traffic along a (non-fixed) link from source to destination. (Throughout, we will be using subscripts for users and superscripts for links.)

Denote $w_{i}$ the traffic of user $i \in[n]$. Define the $n \times 1$ traffic vector $\mathbf{w}$ in the natural way. Assume throughout that $m>1$ and $n>1$. Assume also, without loss of generality, that $w_{1} \geq w_{2} \geq \ldots \geq w_{n}$. Denote $W=\sum_{i \in[n]} w_{i}$.

A pure strategy for user $i \in[n]$ is some specific link. A mixed strategy for user $i \in[n]$ is a probability distribution over pure strategies; thus, a mixed strategy is a probability distribution over the set of links. The support of the mixed strategy for user $i \in[n]$, denoted $\sup (i)$, is the set of those pure strategies (links) to which $i$ assigns positive probability. A pure strategy profile is represented by an $n$-tuple $\left\langle\ell_{1}, \ell_{2}, \ldots, \ell_{n}\right\rangle \in[m]^{n}$; a mixed strategy profile is represented by an $n \times m$ probability matrix $\mathbf{P}$ of $n m$ probabilities $p_{i}^{j}, i \in[n]$ and $j \in[m]$, where $p_{i}^{j}$ is the probability that user $i$ chooses link $j$. 
For a probability matrix $\mathbf{P}$, define indicator variables $I_{i}^{j} \in\{0,1\}$, where $i \in[n]$ and $j \in[m]$, such that $I_{i}^{j}=1$ if and only if $p_{i}^{j}>0$. Thus, the support of the mixed strategy for user $i \in[n]$ is the set $\left\{j \in[m] \mid I_{i}^{j}=1\right\}$. For each link $j \in[m]$, define the view of link $j$, denoted view $(j)$, as the set of users $i \in[n]$ that potentially assign their traffics to link $j$; so, view $(j)=\left\{i \in[n] \mid I_{i}^{j}=1\right\}$. For each link $j \in[m]$, denote $V^{j}=\mid$ view $(j) \mid$.

A mixed strategy profile $\mathbf{P}$ is fully mixed [22, Section 2.2] if for all users $i \in[n]$ and links $j \in[m], I_{i}^{j}=1$. Throughout, we will cast a pure strategy profile as a special case of a mixed strategy profile in which all (mixed) strategies are pure.

\subsection{System, Models, and Cost Measures}

Denote $c^{\ell}>0$ the capacity of link $\ell \in[m]$, representing the rate at which the link processes traffic. So, the latency for traffic $w$ through link $\ell$ equals $w / c^{\ell}$. In the model of uniform capacities, all link capacities are equal to $c$, for some constant $c>0$; link capacities may vary arbitrarily in the model of arbitrary capacities. Assume throughout, without loss of generality, that $c^{1} \geq c^{2} \geq \ldots \geq c^{m}$. Denote $C=\sum_{j \in[m]} c^{j}$. In the model of identical traffics, all user traffics are equal to 1 ; user traffics may vary arbitrarily in the model of arbitrary traffics.

For a pure strategy profile $\left\langle\ell_{1}, \ell_{2}, \ldots, \ell_{n}\right\rangle$, the latency cost for user $i \in[n]$, denoted $\lambda_{i}$, is $\left(\sum_{k: \ell_{k}=\ell_{i}} w_{k}\right) / c^{\ell_{i}}$; that is, the latency cost for user $i$ is the latency of the link it chooses. For a mixed strategy profile $\mathbf{P}$, denote $\delta^{\ell}$ the actual traffic on link $\ell \in[m]$; so, $\delta^{\ell}$ is a random variable. For each link $\ell \in[m]$, denote $\theta^{\ell}$ the expected traffic on link $\ell \in[m]$; thus, $\theta^{\ell}=\mathcal{E}\left(\delta^{\ell}\right)=\sum_{i=1}^{n} p_{i}^{\ell} w_{i}$. Given $\mathbf{P}$, define the $m \times 1$ expected traffic vector $\Theta$ induced by $\mathbf{P}$ in the natural way. Given $\mathbf{P}$, denote $\Lambda^{\ell}$ the expected latency on link $\ell \in[m]$; clearly, $\Lambda^{\ell}=\frac{\theta^{\ell}}{c^{\ell}}$. Define the $m \times 1$ expected latency vector $\Lambda$ in the natural way. For a mixed strategy profile $\mathbf{P}$, the expected latency cost for user $i \in[n]$ on link $\ell \in[m]$, denoted $\lambda_{i}^{\ell}$, is the expectation, over all random choices of the remaining users, of the latency cost for user $i$ had its traffic been assigned to link $\ell$; thus,

$$
\lambda_{i}^{\ell}=\frac{w_{i}+\sum_{k=1, k \neq i} p_{k}^{\ell} w_{k}}{c^{\ell}}=\frac{\left(1-p_{i}^{\ell}\right) w_{i}+\theta^{\ell}}{c^{\ell}} .
$$

For each user $i \in[n]$, the minimum expected latency cost, denoted $\lambda_{i}$, is the minimum, over all links $\ell \in[m]$, of the expected latency cost for user $i$ on link $\ell$; thus, $\lambda_{i}=\min _{\ell \in[m]} \lambda_{i}^{\ell}$. For a probability matrix $\mathbf{P}$, define the $n \times 1$ minimum expected latency cost vector $\lambda$ induced by $\mathbf{P}$ in the natural way.

Associated with a traffic vector $\mathbf{w}$, a capacity vector $\mathbf{c}$ and a mixed strategy profile $\mathbf{P}$ is the Quadratic Social Cost, denoted QSC $(\mathbf{w}, \mathbf{c}, \mathbf{P})$, which is the expectation of the sum of squares of the incurred link latencies; thus,

$$
\begin{aligned}
\operatorname{QSC}(\mathbf{w}, \mathbf{c}, \mathbf{P}) & =\mathcal{E}\left(\sum_{\ell \in[m]} \frac{\left(\sum_{k: \ell_{k}=\ell} w_{k}\right)^{2}}{c^{\ell}}\right) \\
& =\sum_{\left\langle\ell_{1}, \ell_{2}, \ldots, \ell_{n}\right\rangle \in[m]^{n}}\left(\prod_{k=1}^{n} p_{k}^{\ell_{k}} \cdot \sum_{\ell \in[m]} \frac{\left(\sum_{k: \ell_{k}=\ell} w_{k}\right)^{2}}{c^{\ell}}\right) .
\end{aligned}
$$


Since the expectation of a sum is equal to the sum of expectations, we can write

$$
\operatorname{QSC}(\mathbf{w}, \mathbf{c}, \mathbf{P})=\sum_{\ell \in[m]} \sum_{A \subset[n]}\left(\prod_{i \in A} p_{i}^{\ell}\right)\left(\prod_{i \notin A}\left(1-p_{i}^{\ell}\right)\right) \frac{\left(\sum_{k: \ell_{k}=\ell} w_{k}\right)^{2}}{c^{\ell}}
$$

The Quadratic Optimum associated with a traffic vector w and a capacity vector c, denoted QOPT $(\mathbf{w}, \mathbf{c})$, is the least possible sum of squares of the incurred link latencies. Note that while QSC $(\mathbf{w}, \mathbf{c}, \mathbf{P})$ is defined in relation to a mixed strategy profile $\mathbf{P}, \mathrm{QOPT}(\mathbf{w}, \mathbf{c})$ refers to the optimum pure strategy profile.

The Maximum Social Cost, denoted $\operatorname{MSC}(\mathbf{w}, \mathbf{c}, \mathbf{P})$, which is used in the original KP model, is defined as the expectation of the maximum of the incurred link latencies. Correspondingly, the Maximum Optimum, denoted MOPT(w, c), is the minimum, over all assignments, maximum incurred link latency.

\subsection{Nash Equilibria}

We are interested in a special class of mixed strategies called Nash equilibria [24] that we describe below. Formally, the probability matrix $\mathbf{P}$ is a Nash equilibrium [19, Section 2] if for all users $i \in[n]$ and links $\ell \in[m], \lambda_{i}^{\ell}=\lambda_{i}$ if $I_{i}^{\ell}=1$, and $\lambda_{i}^{\ell}>\lambda_{i}$ if $I_{i}^{\ell}=0$. Thus, each user assigns its traffic with positive probability only on links (possibly more than one of them) for which its expected latency cost is minimized; this implies that there is no incentive for a user to unilaterally deviate from its mixed strategy in order to avoid links on which its expected latency cost is higher than necessary.

Mavronicolas and Spirakis [22, Lemma 15] show that in the model of arbitrary users and identical links, all links are equiprobable in a fully mixed Nash equilibrium.

Lemma 3 (Mavronicolas and Spirakis [22]). Consider the model of arbitrary users and identical links. Then, there exists a unique fully mixed Nash equilibrium with associated Nash probabilities $p_{i}^{\ell}=1 / m$, for any user $i \in[n]$ and link $\ell \in[m]$.

\subsection{Coordination Ratio and Quadratic Coordination Ratio}

The Quadratic Coordination Ratio is the maximum value, over all traffic vectors $\mathbf{w}$, capacity vectors $\mathbf{c}$, and Nash equilibria $\mathbf{P}$ of the ratio $\frac{\mathrm{QSC}(\mathbf{w}, \mathbf{c}, \mathbf{P})}{\mathrm{QOPT}(\mathbf{w}, \mathbf{c})}$. In a corresponding way, the Maximum Coordination Ratio is defined in [19] as the maximum value, over all traffic vectors $\mathbf{w}$, capacity vectors $\mathbf{c}$ and Nash equilibria $\mathbf{P}$ of the ratio $\frac{\mathrm{MSC}(\mathbf{w}, \mathbf{c}, \mathbf{P})}{\operatorname{MOPT}(\mathbf{w}, \mathbf{c})}$.

\section{The Quadratic Social Cost of Nash Equilibria}

In this section, we study the Quadratic Social Cost of arbitrary (mixed) Nash equilibria. We start by proving: 
Theorem 1 (Quadratic Social Cost of Arbitrary Nash Equilibrium). Fix any traffic vector $\mathbf{w}$, any capacity vector $\mathbf{c}$, and any arbitrary Nash equilibrium $\mathbf{P}$. Then, QSC $(\mathbf{w}, \mathbf{c}, \mathbf{P})$ can be computed in time $O(n m W)$.

We next establish that the Quadratic Social Cost takes a particularly nice form for the case of the fully mixed Nash equilibrium. We prove:

Theorem 2 (Quadratic Social Cost of Fully Mixed Nash Equilibrium). Consider the model of arbitrary users and identical links. Then, for any traffic vector $\mathbf{w}$,

$$
\mathrm{QSC}(\mathbf{w}, \mathbf{F})=W_{1}+\frac{2}{m} W_{2},
$$

where $W_{1}=\sum_{i \in[n]} w_{i}^{2}$ and $W_{2}=\sum_{i, k \in[n], i \neq k} w_{i} w_{k}$.

The next Lemma is used in the proof of Proposition 1 .

Lemma 4. Let $a, n \in \mathbb{N}$, a even, let $p_{i} \in[0,1]$ for all $1 \leq i \leq n$. Denote $P=\left(p_{1}, \ldots, p_{n}\right)$ and $p=\sum_{1 \leq i \leq n} p_{i}$. Set

$$
H(P)=\sum_{A \subset[n]}|A|^{a}\left\{\prod_{i \in A} p_{i}\right\}\left\{\prod_{j \notin A}\left(1-p_{j}\right)\right\} .
$$

Define $\tilde{P}$ by $\tilde{p}_{i}=\frac{1}{n} \cdot p$ for all $1 \leq i \leq n$. Then $H(P) \leq H(\tilde{P})$.

Proposition 1. Consider the model of identical users and identical links. Then, for any arbitrary Nash equilibrium $\mathbf{P}$,

$$
\mathrm{QSC}(\mathbf{P}) \leq \sum_{j \in[m]}\left(\theta^{j}+\frac{r_{j}-1}{r_{j}}\left(\theta^{j}\right)^{2}\right),
$$

where $\theta^{j}=\sum_{i \in[n]} p_{i}^{j}$ and $r_{j}=|\operatorname{view}(j)|$.

We finally prove:

Theorem 3. Consider the model of identical users and related links. Then,

$$
\mathrm{QSC}(\mathbf{c}, \mathbf{F})=\frac{n(n+m-1)}{C} .
$$

Corollary 1 (Quadratic Social Cost of Fully Mixed Nash Equilibrium). Consider the model of identical users and identical links. Then,

$$
\mathrm{QSC}(\mathbf{w}, \mathbf{c}, \mathbf{F})=\frac{n(n+m-1)}{m} .
$$




\section{The Worst-Case Nash Equilibrium}

We now establish that, for the model of identical users and identical links, the worst-case Nash equilibrium is the fully mixed Nash equilibrium. We start our proof with a technical lemma which holds in the more general model of arbitrary users, and then return to the model of identical users and identical links.

Lemma 5. Consider $n$ arbitrary users on $m$ identical links, and let $j, k \in[m]$.

1. If view $(j)=\operatorname{view}(k) \neq \emptyset$, then $|\operatorname{view}(j)|=1$ or $\theta^{j}=\theta^{k}$ and $p_{i}^{j}=p_{i}^{k}$ for all $i \in[n]$.

2. If view $(j) \subsetneq \operatorname{view}(k)$, then $\theta^{j}>\theta^{k}$.

Theorem 4. Consider the model of identical users and identical links. Then, for any arbitrary Nash equilibrium $\mathbf{P}, \mathrm{QSC}(\mathbf{w}, \mathbf{c}, \mathbf{P}) \leq \mathrm{QSC}(\mathbf{w}, \mathbf{c}, \mathbf{F})$.

\section{Bounds on Quadratic Coordination Ratio}

In this section, we present our bounds on Quadratic Coordination Ratio. We start by proving:

Theorem 5 (Quadratic Coordination Ratio for Pure Nash Equilibria). Consider the model of arbitrary users and identical links, restricted to pure Nash equilibria. Then,

$$
\max _{\mathbf{w}, \mathbf{P}} \frac{\operatorname{QSC}(\mathbf{w}, \mathbf{P})}{\operatorname{QOPT}(\mathbf{w})}=\frac{9}{8} .
$$

We give here only a sketch of the proof. Let there be $n$ users and $m$ links. If $n \leq m$, then every pure Nash equilibrium has optimal social cost. Now assume $n>m$. Let $\mathbf{P}$ be any pure Nash equilibrium. Let us first assume that $w_{i} \leq \frac{W}{m}$ holds for all users $i \in[n]$. Let $B=\min _{j \in[m]} \delta^{j}$ be the minimum traffic on any of the links. Then $B>0$, and it has been shown in [17, that on every link the load is bounded by $2 B$.

We use some iterative procedure to compute an upper bound for QSC $(\mathbf{w}, \mathbf{c}, \mathbf{P})$. When the algorithm terminates, then we know that there exists some $k \in[m]$, such that

$-\mathrm{QSC}(\mathbf{w}, \mathbf{c}, \mathbf{P}) \leq \sum_{j \in[m]} x_{j}^{2}$,

$-x_{j}=2 B$ for $k$ links,

$-x_{j}=B+x, 0 \leq x \leq B$, for one link, and

$-x_{j}=B$ for $m-k-1$ links.

Note, that QOPT $(\mathbf{w}, \mathbf{c}) \geq \frac{W 2}{m}$, and therefore

$$
\frac{\operatorname{QSC}(\mathbf{w}, \mathbf{c}, \mathbf{P})}{\operatorname{QOPT}(\mathbf{w}, \mathbf{c})} \leq \frac{((3 k+m-1) B 2+(B+x) 2) m}{(m B+k B+x) 2}=f(k) \text {. }
$$


Maximizing the function $f(k)$ shows the upper bound for the case that $w_{i} \leq \frac{W}{m}$ for all $i \in[n]$.

In case that $w_{i}>\frac{W}{m}$ holds for some user $i \in[n]$, such a user is alone on its link in every Nash equilibrium $\mathbf{P}$, and both the user and the link can be omitted, increasing the coordination ratio. To show tighness, we give an instance.

We continue by a similar result for the reciprocal case of identical users and related links:

Theorem 6 (Quadratic Coordination Ratio for Pure Nash Equilibria). Consider the model of identical users and related links, restricted to pure Nash equilibria. Then,

$$
\max _{\mathbf{w}, \mathbf{c}, \mathbf{P}} \frac{\operatorname{QSC}(\mathbf{w}, \mathbf{c}, \mathbf{P})}{\operatorname{QOPT}(\mathbf{w}, \mathbf{c})}=\frac{4}{3}
$$

We give here only a sketch of the full proof. First, we show that no instance with traffic vector $\mathbf{w}=\{1\}^{n}$, capacity vector $\mathbf{c}$ and pure Nash equilibrium $\mathbf{P}$ exists with Quadratic Coordination Ratio greater than $\frac{4}{3}$. Therefore, we assume, by way of contradiction, that such an instance exists, and fix the minimal (in the number of links) such counterexample, its worst case Nash equilibrium $\mathbf{P}$ and an optimal assignment $\mathbf{Q}$. We denote the traffic of each link $j$ by $\delta^{j}(\mathbf{P})$ when referring to $\mathbf{P}$, and by $\delta^{j}(\mathbf{Q})$ when referring to the optimum assignment. Lemma 6] shows, that $\delta^{j}(\mathbf{P})$ is at most by one smaller than $\delta^{j}(\mathbf{Q})$ for any link $j$. Lemma 7 shows, that for the instance under consideration, only for exactly one link $k$, $\delta^{k}(\mathbf{P})$ is greater than $\delta^{k}(\mathbf{Q})$, and that no link has the same traffic according to $\mathbf{P}$ and $\mathbf{Q}$. This implies $\delta^{k}(\mathbf{P})=\delta^{k}(\mathbf{Q})+m-1$, because all remaining links must have $\delta^{j}(\mathbf{P})=\delta^{j}(\mathbf{Q})-1$. Lemma 8 shows, that, if not all links except for $k$ have the same capacity $c^{j}$ and the same traffic, then we can create a new instance with $m-1$ identical links, having the same traffic, and one additional link, which has at least the same Quadratic Coordination Ratio. Hence, we can consider this new instance in order to bound the Quadratic Coordination Ratio of the original instance from above. To do so, we write down an optimization problem which overestimates the Quadratic Coordination Ratio of the new instance and includes, as constraints, the Nash equilibrium property and optimality criterion from Lemma 9. The optimization problem evaluates to $\frac{4}{3}$, which contradicts the initial assumption.

To proof that the bound is tight, we construct an instance with Quadratic Coordination Ratio $\frac{4}{3}$ for any number of links $m$.

Lemma 6. Let $(\mathbf{w}, \mathbf{c}), \mathbf{Q}, \mathbf{P}, \delta^{j}(\mathbf{Q})$ and $\delta^{j}(\mathbf{P})$ be as in the proof of Theorem 6 . Then, $\delta^{j}(\mathbf{Q})-\delta^{j}(\mathbf{P}) \leq 1$ for all $j \in[m]$.

Lemma 7. Let $(\mathbf{w}, \mathbf{c}), \mathbf{Q}, \mathbf{P}, \delta^{j}(\mathbf{Q})$ and $\delta^{j}(\mathbf{P})$ be as in the proof of Theorem 6 ] Then, $\delta^{k}(\mathbf{P})=\delta^{k}(\mathbf{Q})+m-1$ for some $k \in[m]$, and $\delta^{j}(\mathbf{P})=\delta^{j}(\mathbf{Q})-1$ for all $j \in[m] \backslash\{k\}$. 
Lemma 8. Let $(\mathbf{w}, \mathbf{c}), \mathbf{Q}, \mathbf{P}, \delta^{j}(\mathbf{Q})$ and $\delta^{j}(\mathbf{P})$ be as in the proof of Theorem 6. Then, there exists an instance $(\tilde{\mathbf{w}}, \tilde{\mathbf{c}})$ that has the same number $m$ of links as $(\mathbf{w}, \mathbf{c})$ with optimal assignment $\tilde{\mathbf{Q}}$, Nash equilibrium assignment $\tilde{\mathbf{P}}$, such that $\delta^{k}(\tilde{\mathbf{P}})=\delta^{k}(\tilde{\mathbf{Q}})+m-1$ for some link $k \in[m], \delta^{i}(\tilde{\mathbf{Q}})=\delta^{j}(\tilde{\mathbf{Q}})$ and $\tilde{c_{i}}=\tilde{c_{j}}$ for all $i, j \in[m] \backslash\{k\}$ and $\frac{\mathrm{QSC}(\tilde{\mathbf{w}}, \tilde{\mathbf{c}}, \tilde{\mathbf{P}})}{\operatorname{QOPT}(\tilde{\mathbf{w}}, \tilde{\mathbf{c}})} \geq \frac{\mathrm{QSC}(\mathbf{w}, \mathbf{c}, \mathbf{P})}{\operatorname{QOPT}(\mathbf{w}, \mathbf{c})}$.

Lemma 9. Let $\mathbf{Q}$ be any pure assignment for an instance $(\mathbf{w}, \mathbf{c})$ of the model of identical traffics and related links, let $\mathbf{w}=(w, \ldots, w)$. Then, $\mathbf{Q}$ is optimal, i.e., $\mathrm{QSC}(\mathbf{w}, \mathbf{c}, \mathbf{Q})=\mathrm{QOPT}(\mathbf{w}, \mathbf{c})$, if and only if for every pair of links $i, j \in[\mathrm{m}]$

$$
\frac{\left(\delta^{i}(\mathbf{Q})+w\right)^{2}}{c_{i}}+\frac{\left(\delta^{j}(\mathbf{Q})-w\right)^{2}}{c_{j}} \geq \frac{\left(\delta^{i}(\mathbf{Q})\right)^{2}}{c_{i}}+\frac{\left(\delta^{j}(\mathbf{Q})\right)^{2}}{c_{j}}
$$

We next prove:

Theorem 7. Consider the model of arbitrary users and identical links. Then,

$$
\max _{\mathbf{w}, \mathbf{c}} \frac{\operatorname{QSC}(\mathbf{w}, \mathbf{c}, \mathbf{F})}{\operatorname{QOPT}(\mathbf{w}, \mathbf{c})} \leq 2-\frac{1}{m} \text {. }
$$

We next prove:

Theorem 8. Consider the model of identical users and identical links. Then, for any traffic vector $\mathbf{w}$, capacity vector $\mathbf{c}$ and mixed Nash equilibrium $\mathbf{P}$,

$$
\frac{\operatorname{QSC}(\mathbf{w}, \mathbf{c}, \mathbf{P})}{\operatorname{QOPT}(\mathbf{w}, \mathbf{c})} \leq 1+\min \left\{\frac{m-1}{n}, \frac{n-1}{m}\right\} \leq 2-\frac{1}{m} .
$$

Acknowledgments. We thank Rainer Feldmann and Martin Gairing for several helpful discussions.

\section{References}

1. N. Alon, Y. Azar, G. J. Woeginger and T. Yadid, Approximation Schemes for Scheduling, Proc. of SODA 1997, pp. 493-500.

2. E. Anshelevich, A. Dasgupta, É. Tardos, and T. Wexler, Near-Optimal Network Design with Selfish Agents, Proc. of STOC 2003, pp. 511-520.

3. M. J. Beckmann, On the Theory of Traffic Flow in Networks, Traffic Quart, Vol. 21, pp. 109-116, 1967.

4. M. Beckmann, C. B. McGuire and C. B. Winsten, Studies in the Economics of Transportation, Yale University Press, 1956.

5. D. Braess, Über ein Paradoxen aus der Verkehrsplanung, Unternehmensforschung, Vol. 12, pp. 258-268, 1968.

6. A. K. Chandra and C. K. Wong, Worst-case Analysis of a Placement Algorithm Related to Storage Allocation, SICOMP 1975, Vol. 4, pp. 249-263.

7. E. Altman, T. Basar, T. Jimenez and N. Shimkin, Competitive Routing in Networks with Polynomial Costs, IEEE Transactions on Automatic Control, Vol. 47, pp. 92-96, 2002. 
8. R. A. Cody and E. G. Coffman, Jr., Record Allocation for Minimizing Expected Retrieval Costs on Crum-Like Storage Devices, JACM, Vol. 23, pp. 103-115, 1976.

9. A. Czumaj and B. Vöcking, Tight Bounds for Worst-Case Equilibria, Proc. of SODA 2002, pp. 413-420.

10. A. Czumaj, P. Krysta and B. Vöcking, Selfish Traffic Allocation for Server Farms, Proc. of STOC 2002, pp. 287-296.

11. S. C. Dafermos and F. T. Sparrow, The Traffic Assignment Problem for a General Network. Journal of Research of the National Bureau of Standards, Series B, Vol. 73B, pp. 91-118, 1969.

12. E. Even-Dar, A. Kesselman and Y. Mansour, "Convergence Time to Nash Equilibria," Proc. of ICALP 2003, J. C. M. Baeten, J. K. Lenstra, J. Parrow and G. J. Woeginger eds., Vol. 2719, pp. 502-513.

13. A. Fabrikant, A. Luthra, E. Maneva, C. H. Papadimitriou, and S. Shenker, On a Network Creation Game, Proc. of PODC 2003, pp. 347-351.

14. R. Feldmann, M. Gairing, T. Lücking, B. Monien and M. Rode, Nashification and the Coordination Ratio for a Selfish Routing Game, Proc. of ICALP 2003, Vol. 2719, pp. 514-526.

15. R. Feldmann, M. Gairing, T. Lücking, B. Monien and M. Rode, Selfish Routing in Non-Cooperative Networks: A Survey, Proc. of MFCS 2003, Vol. 2747, pp. 21-45.

16. D. Fotakis, S. Kontogiannis, E. Koutsoupias, M. Mavronicolas and P. Spirakis, The Structure and Complexity of Nash Equilibria for a Selfish Routing Game, Proc. of ICALP 2002, Vol. 2380, pp. 123-134.

17. M. Gairing, T. Lücking, M. Mavronicolas, B. Monien and P. Spirakis, The Structure and Complexity of Extreme Nash Equilibria, submitted for publication, 2003.

18. E. Koutsoupias, M. Mavronicolas and P. Spirakis, Approximate Equilibria and Ball Fusion, Proc. of SIROCCO 2002, pp. 223-235.

19. E. Koutsoupias and C. H. Papadimitriou, Worst-case Equilibria, Proc. of STACS 1999, G. Meinel and S. Tison eds., Vol. 1563, pp. 404-413.

20. J. Y. T. Leung and W. D. Wei, Tighter Bounds on a Heuristic for a Partition Problem, Information Processing Letters, Vol. 56, pp. 51-57, 1995.

21. T. Lücking, M. Mavronicolas, B. Monien, M. Rode, P. Spirakis and I. Vrto, Which is the Worst-case Nash equilibrium?, Proc. of MFCS 2003, B. Rovan and P. Vojtas eds., Vol. 2747, pp. 551-561.

22. M. Mavronicolas and P. Spirakis, The Price of Selfish Routing, Proc. of STOC 2001, pp. 510-519.

23. J. F. Nash, Equilibrium Points in $N$-Person Games, Proceedings of the National Academy of Sciences, Vol. 36, pp. 48-49, 1950.

24. J. F. Nash, Non-cooperative Games, Annals of Mathematics, Vol. 54, No. 2, pp. 286-295, 1951.

25. M. J. Osborne and A. Rubinstein, A Course in Game Theory, The MIT Press, 1994.

26. C. H. Papadimitriou, Algorithms, Games and the Internet, Proc. of STOC 2001, pp. 749-753.

27. T. Roughgarden, The Price of Anarchy is Independent of the Network Topology, Proc. of STOC 2002, pp. 428-437.

28. T. Roughgarden, Selfish Routing, Ph. D. Thesis, Department of Computer Science, Cornell University, May 2002.

29. T. Roughgarden and É. Tardos, How Bad is Selfish Routing? JACM, Vol. 49, pp. 236-259, 2002.

30. J. G. Wardrop. Some Theoretical Aspects of Road Traffic Research, Proceedings of the of the Institute of Civil Engineers, Pt. II, Vol. 1, pp. 325-378, 1952. 